\title{
SLOTTED-IRIS STRUCTURE STUDIES
}

\author{
E. Jensen, I. Syratchev, W. Wuensch, CERN, Geneva, Switzerland
}

\begin{abstract}
Accelerating structures with strong transverse-mode damping are required in both the $30 \mathrm{GHz}$ CLIC main linac and the $3 \mathrm{GHz}$ CTF3 drive-beam accelerator. Damping via slotted irises has been investigated for both structures. The transverse wake, the effect of the slots on the fundamental-mode parameters such as $Q$, sensitivity to tolerances, and surface-field enhancements have been computed. Terminating loads have been designed and machining studies to obtain rounded slot edges have been made. A 32-cell prototype $3 \mathrm{GHz}$ structure is being fabricated for the drive beam accelerator of CTF3.
\end{abstract}

\section{INTRODUCTION}

There are a growing number of applications, especially in linear colliders and $\mathrm{B}$-factories, that require accelerating structures in which long-range transverse wakefields are suppressed in order to limit the growth of transverse instabilities in multi-bunch beams. Two techniques, often used together, have been adopted for long-range transverse wakefield suppression: detuning and damping. Detuning functions by varying cell dimensions along the length of a structure in such a way that a frequency spread is induced in higher-order modes without affecting the frequency of the accelerating mode. The transverse wake is suppressed through the decoherence of the fields generated along the length of the structure, but the energy associated with the transverse fields remains within the structure. Damping, on the other hand, functions by absorbing the energy from the transverse fields in a way that limits the absorption of energy from the accelerating mode. Four main techniques have been developed for damping in linear accelerator applications: manifold [1], cut-off waveguide [2], chokemode [3] and slotted iris. Each technique brings with it a different combination of performance potentials, performance limits and fabrication complications.

Slotted iris structures have radial slots in the irises that separate the cells of a disk-loaded waveguide as shown in Figure 1 . The slots function by coupling to the azimuthal currents that are induced in irises by transverse modes and not coupling to the purely radial currents of the (azimuthally symmetric) fundamental mode. The slots lead directly into waveguides that are terminated individually. These waveguides can be double-ridged in cross section, allowing the dipole mode to propagate in a waveguide with a width equal to the cell thickness.

Slotted-iris structures have been proposed for linear colliders already some time ago $[4,5]$, however they have since received much less attention than the other damping techniques. This paper presents some highlights of recent work on slotted-iris structures made in the context of the CLIC study. An attempt has been made to address the most basic, both positive and negative, performance issues of slotted-iris structures in a quantitative way. Among the issues covered are: achievable transversemode $Q$ 's, coupling to low phase advances, slot modes, load design, field enhancement, slot tolerances and coupling to the fundamental.

The work shows that slotted irises can damp the lowest (dominant) transverse mode to a very low $Q$ value, below 20 , with little effect on the fundamental mode $Q$ (which is very desirable also to reduce pulsed surface heating [2]). The relative weaknesses of slotted irises, such as fundamental mode field enhancement and slot tolerances, have been shown to be quite tolerable. The attractiveness of the slotted-iris structure is such that it is being considered for the drive-beam accelerator of CTF3 [6].

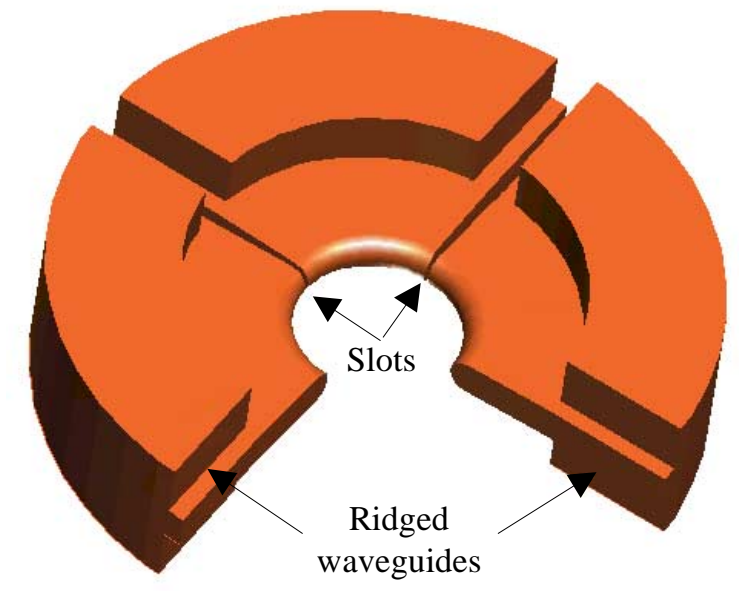

Figure 1: Layout of a single cell of a slotted-iris structure.

\section{TRANSVERSE MODE PERFORMANCE}

$Q$ values are primarily determined by the slot width - a wider slot gives a lower $Q$ - with typical values below 20 .

One limitation to damping with slots is that they do not in general couple to low phase advance modes. For low phase advances, currents on opposite sides of the iris are in opposite directions, giving no net displacement current across the slot. The effect is of limited importance for the lowest dipole bands where primarily high phase advances, typically around $150^{\circ}$, are excited by a speed of light beam. Mainly higher dipole bands, the 4th to the 6th, are excited in lower phase advances. The width of the ridge in the damping waveguide can be set so that the waveguide is propagating in its next higher mode, the $\mathrm{TE}_{02}$, in the frequency range of the higher bands. This mode is excited by the currents of the low phase advance modes. The zero phase advance modes are consequently damped to $Q$ values of typically 100 to 300 . 
Another limitation of slotted iris structures is the appearance of 'slot modes' as shown in Figure 2. These modes have electric fields concentrated in the damping slots and magnetic fields which circle the iris quadrants (or halves) formed by the slots. These modes have a frequency where a quarter of a free-space wavelength is slightly more than the distance from the center of the iris to the cavity wall. The frequency of a slotted-iris mode is about $2 / 3$ of the fundamental-mode frequency and has a loss factor of the order of $5 \%$ of the lowest dipole mode. The slot modes are well damped if the cut-off frequency of the waveguide is chosen to be below the slot-mode frequency.

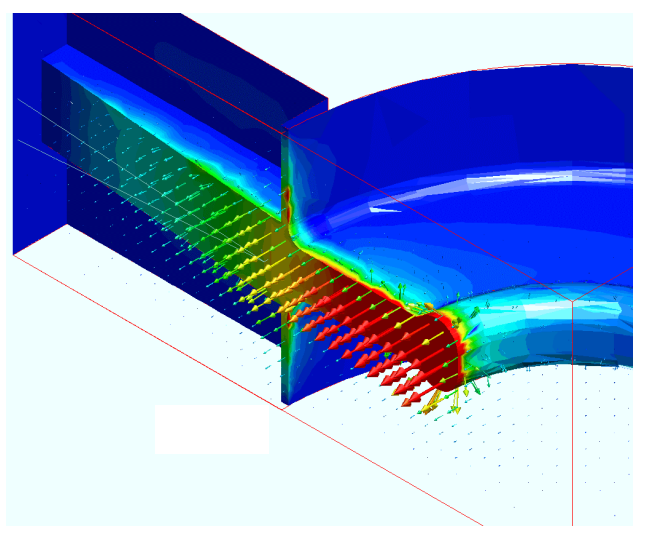

Figure 2: Electric field pattern of a slot mode.

\section{ACCELERATING MODE PERFORMANCE}

One important observation for the design of the coupling slots has been that the edges of slots should be exactly radial rather than be parallel (constant slot width). In this way perturbation of fundamental-mode currents is minimized. A purely radial slot which begins $0.6 \mathrm{~mm}$ wide at the iris in a $3 \mathrm{GHz}$ structure detunes the fundamental mode by less than $3 \mathrm{kHz}$ (the resolution limit of the computation). This is because, to first order, the electric and magnetic stored energy in the slot is equal.

Such a purely radial slot also has very little effect on the fundamental mode $Q$, with an external $Q$ of more than $10^{7}$ for the case just mentioned. Errors in the position of the slots dominate degradation of the fundamental-mode $Q$. The cases where the damping waveguide cut-off frequency is above or below fundamental must be considered separately. When the cut-off is below the fundamental, power flows directly to the load. When it is above, the power is dissipated in the walls of the coupling irises. The results are summarised in Figure 3.

Slotted irises affect the fundamental mode in another important way by creating a region of enhanced surface electric field in the area near the slot and the center of the iris as shown in Figure 4. The field-enhancement factor is a function of the dimensionless constant given by the radius of the junction divided by the width of the slot. The results of the systematic computation of this effect are shown in Figure 5.

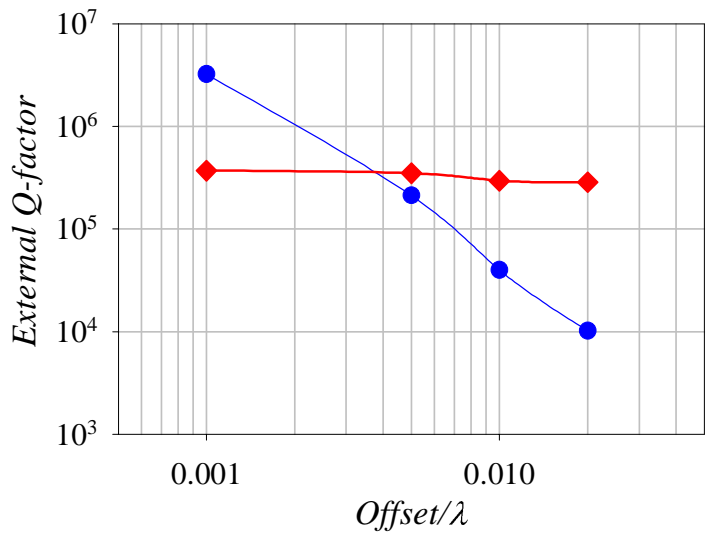

Figure 3: Degradation of fundamental mode $Q$ as a function of slot offset. A fundamental frequency of $30 \mathrm{GHz}$ and a damping waveguide cut-off of $32 \mathrm{GHz}$ gives the red (diamond) curve, and a cut-off of $26 \mathrm{GHz}$ gives the blue (circle) curve.

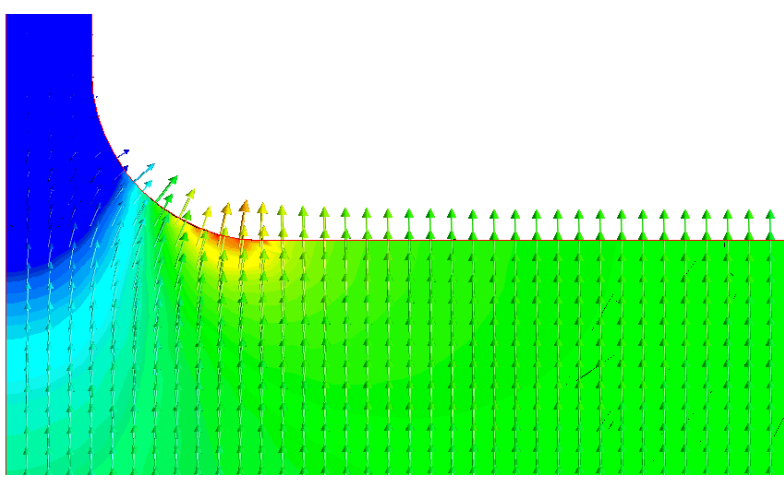

Figure 4: Surface electric field enhancement due to the presence of the slot. The center of the iris is towards the bottom of the picture, and the damping slot extends upwards. The case of a slot/iris edge rounded with a radius equal to the slot width is shown.

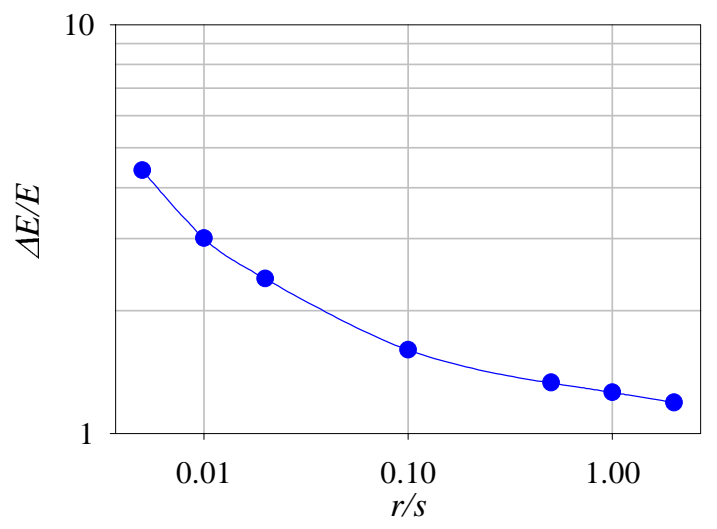

Figure 5: Surface field enhancement factor as a function of the normalised radius on the edge of the slot. 


\section{LOADS AND FABRICATION}

In addition to the $Q$ of the dipole modes, the transverse wakefield of the structure also depends on the quality of the termination of the damping waveguides. As has been calculated for the CLIC TDS (Tapered Damped Structure [2]) a reflection coefficient $|\Gamma|<0.1$ in the frequency range of the lowest dipole mode is sufficient to produce a transverse wake very close to the ideal case of a perfectly terminated damping waveguide. Tapered loads made from silicon carbide that fit inside the ridges of the damping waveguides provide such a performance. At $30 \mathrm{GHz}$ however, these loads become very small - less than $0.1 \mathrm{~mm}$ thick! A lossy extension of the damping waveguides made from silicon carbide and mounted on the outside of the cells provides a more practical and equally performing alternative to internal loads. The layout of such a load is shown in Figure 6.

Solutions for fabrication of the slots themselves are also highly dependent on the frequency of the structures. At $3 \mathrm{GHz}$, damping slots are typically a millimeter wide and require tolerances of the order of $0.01 \mathrm{~mm}$. This is certainly accessible by classical machining techniques. An example is the drive beam accelerator of CTF3, one cell of which is shown in Figure 7. At $30 \mathrm{GHz}$, slotted iris cells made from a single piece appear to be challenging, so a few test cells have been produced to investigate feasibility. The parts shown in Figure 8 are $30 \mathrm{GHz}$ cells with an iris aperture of $4 \mathrm{~mm}$. The part on the left has $0.1 \mathrm{~mm}$ wide slots formed by wire-cutting using electrical-discharge machining. The slots however have sharp edges. The part on the right has $0.25 \mathrm{~mm}$ wide slots with $0.25 \mathrm{~mm}$ radii joining the slots to the iris surface. The slots were milled on a CNC milling machine using a formed mill. The position tolerance on both types of slots is about of $0.01 \mathrm{~mm}$.

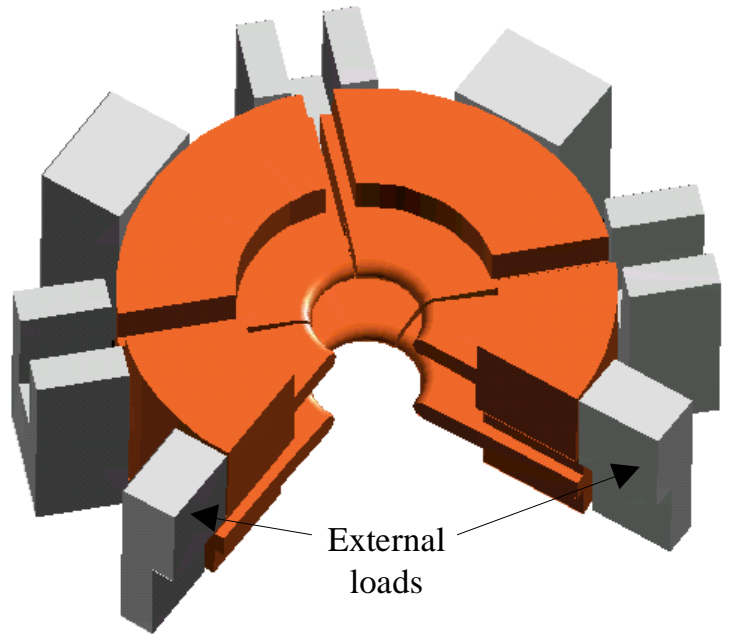

Figure 6: Configuration of an external load.

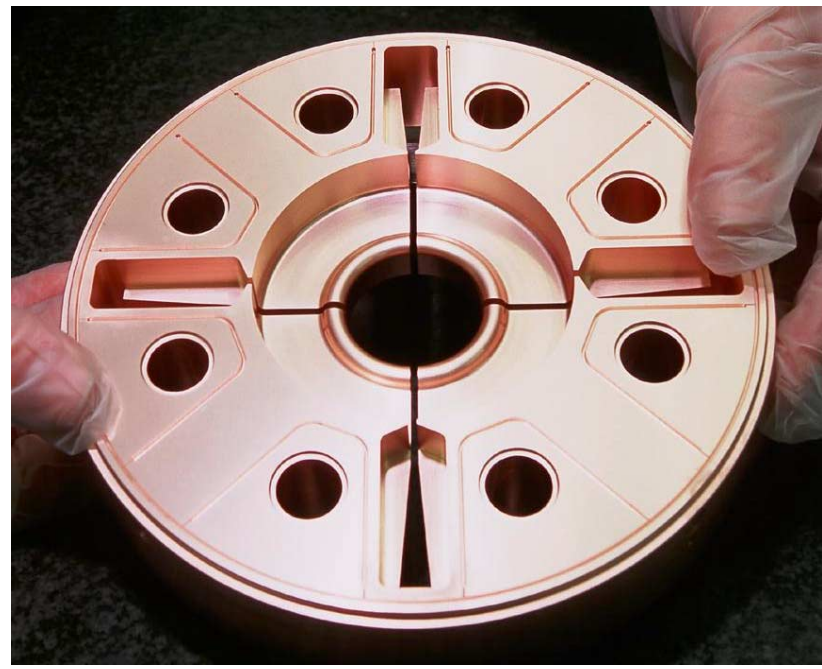

Figure 7: Prototype $3 \mathrm{GHz}$ cell for the CTF3 drive beam accelerator.

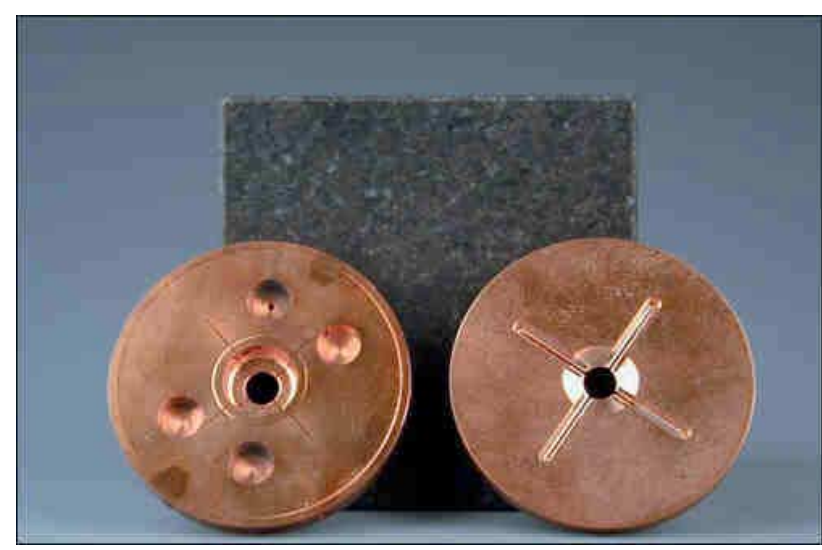

Figure 8: Machining test of $30 \mathrm{GHz}$ slotted iris disks.

\section{REFERENCES}

[1] C. Adolphsen et. al. "Wakefield and Beam Centering Measurements of a Damped and Detuned X-Band Accelerator Structure", PAC'99, New York, March 1999. [2] I. Wilson, W. Wuensch, "The CLIC Main Linac Accelerating Structure," LINAC2000, Monterey, California, August 2000.

[3] N. Akasaka, T. Shintake, H. Matsumoto, "Optimization on Wakefield Damping in C-Band Accelerating Structure," LINAC98, Chicago, August 1998.

[4] W. Schnell, "New Developments in Accelerator Technology," Europhysics Conference on High Energy Physics, Madrid, September 1989.

[5] H. Deruyter et. al., "Damped and Detuned Accelerator Structures," SLAC Pub. 5322, 1990.

[6] H.Braun, "Achievements and Future Plans of CLIC Test Facilities,” HEACC2001, Tsuukba, March 2001. 